معادلة التكامل المشترك والتباطؤ الزمني لقياس العلاقة بين الناتج الزراعي والناتج المحلي الإجمالي

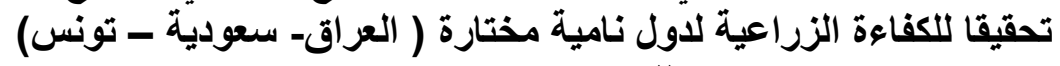

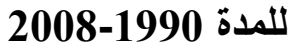

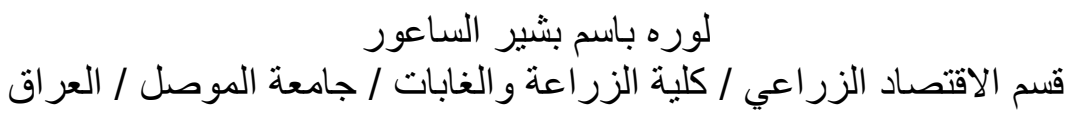

E-mail: Lora.basim@yahoo.com

\section{الخلاصة}

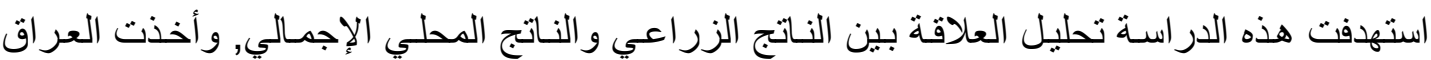
و السعودية وتونس كحالة تطبيقية, و اعتمد البحث على بيانات سنوية خـلال المدة (1990-2008)، وتنـاول

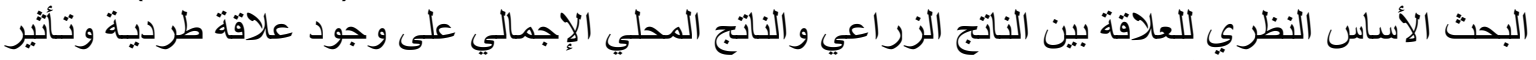

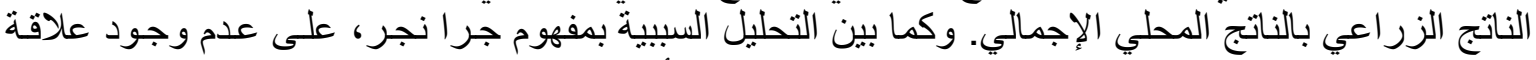

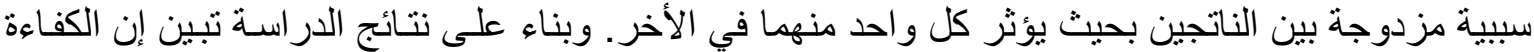

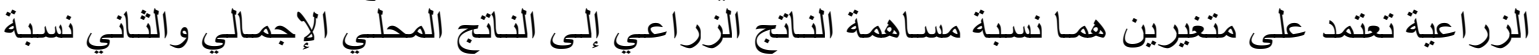

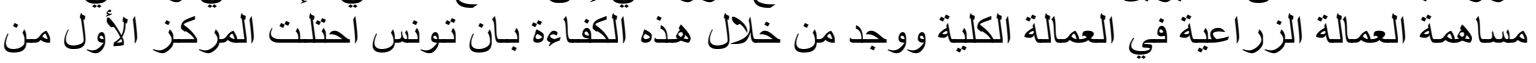

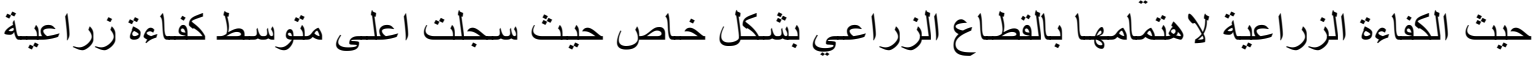

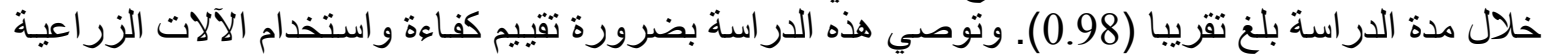

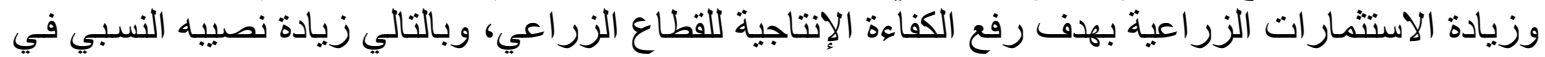

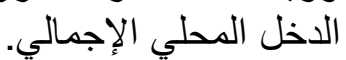
كلمات دالة : التكامل المشترك الإكي Agricultural Efficiency ، الناتج المحلي الإجمالي.

\section{المقدمة}

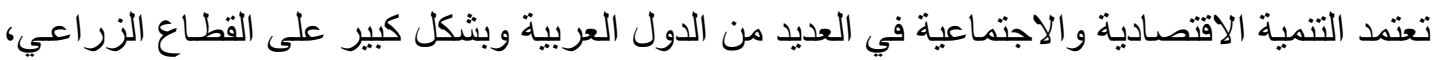

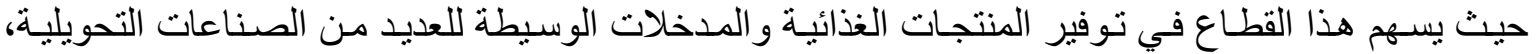

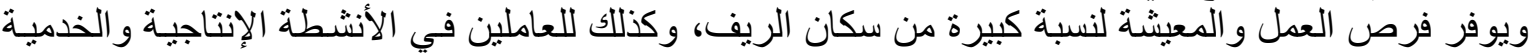

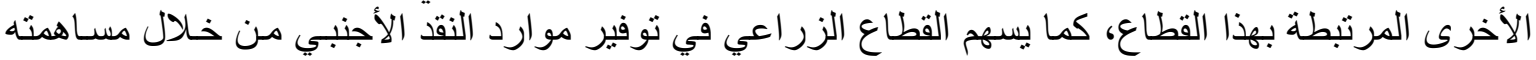

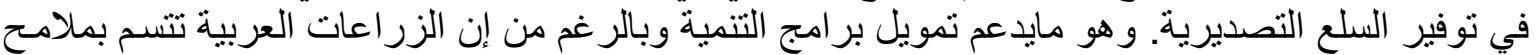

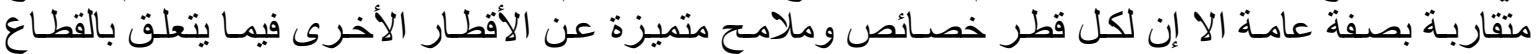

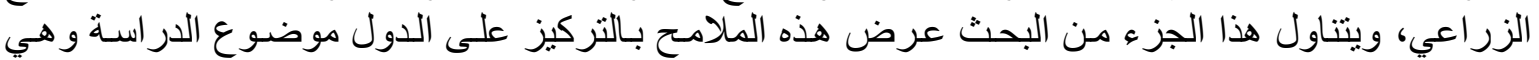

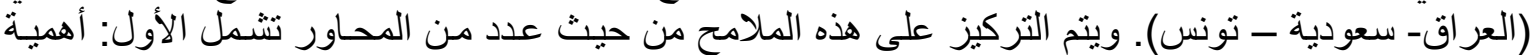

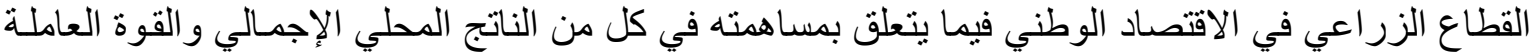

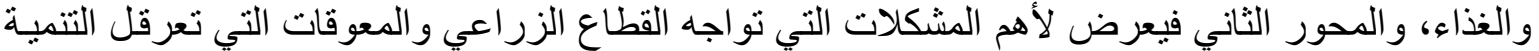

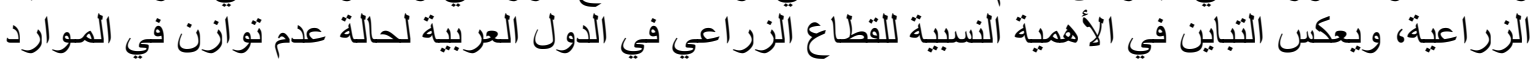

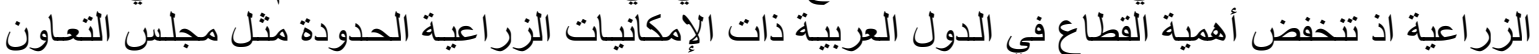

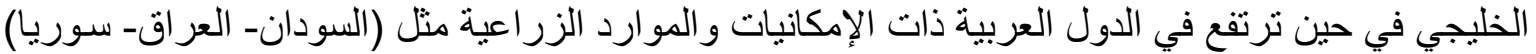

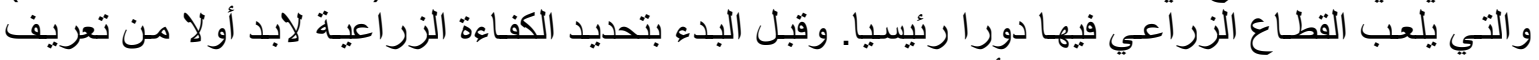

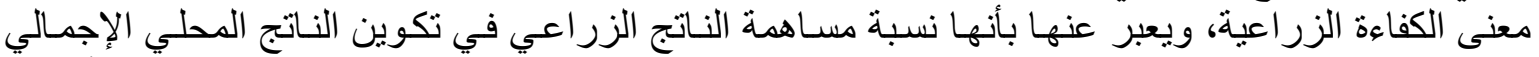

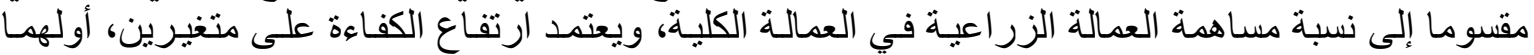

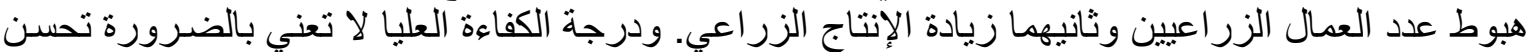

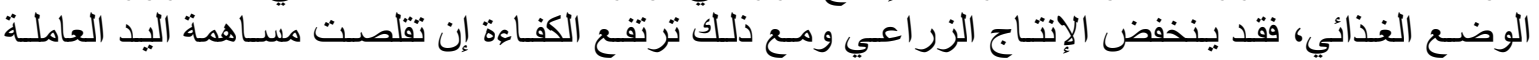

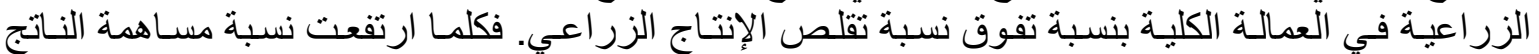

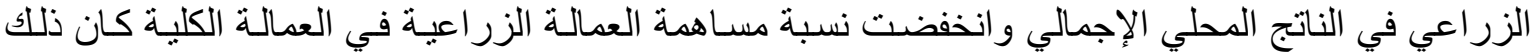

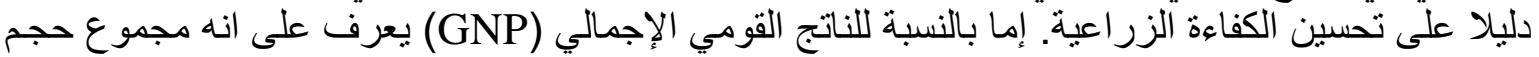

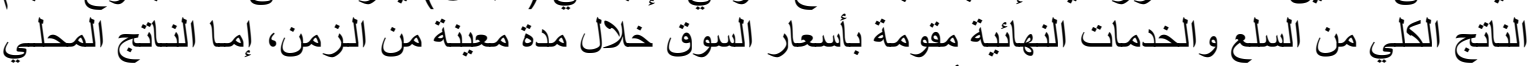

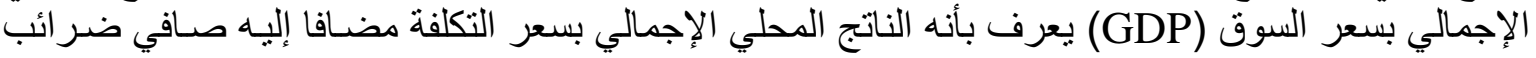




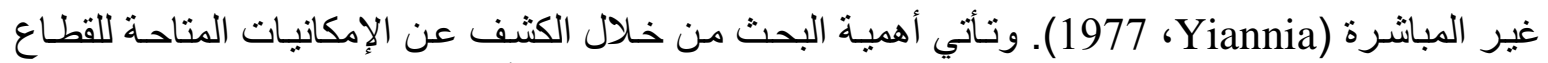

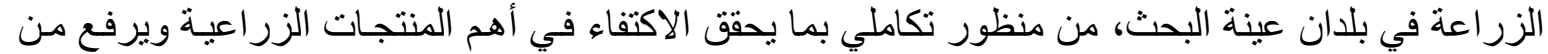

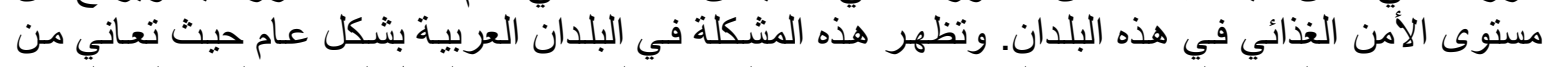

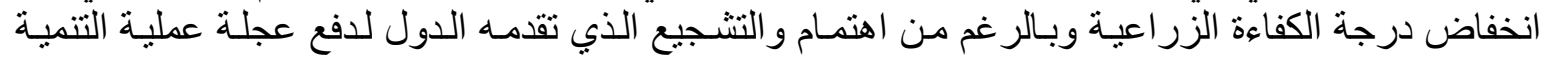

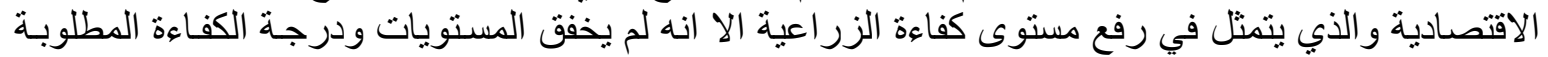

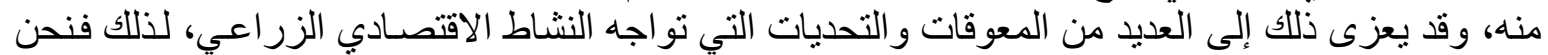

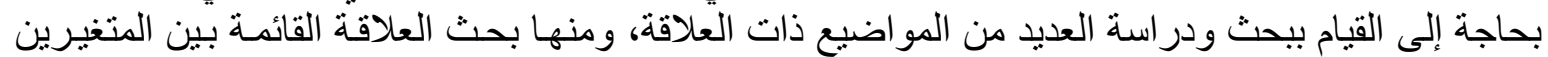

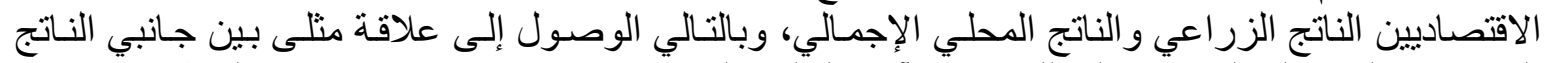

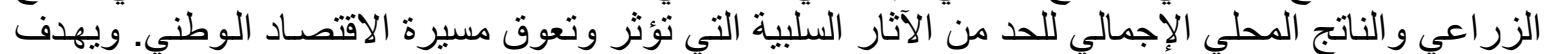

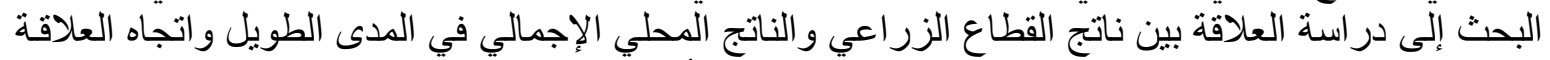

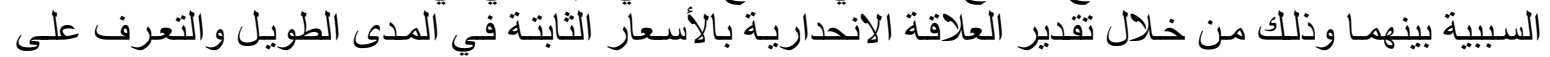

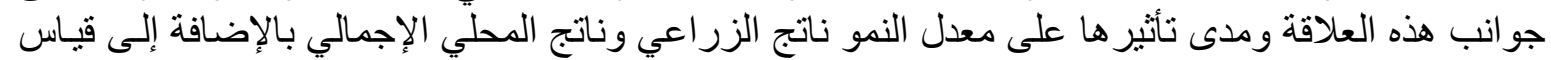

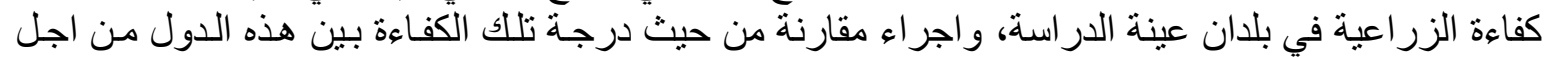

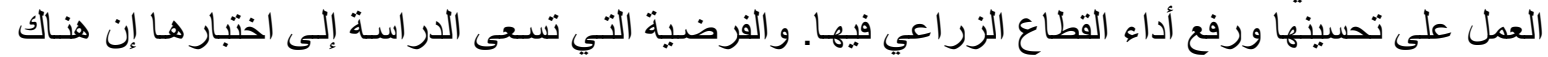

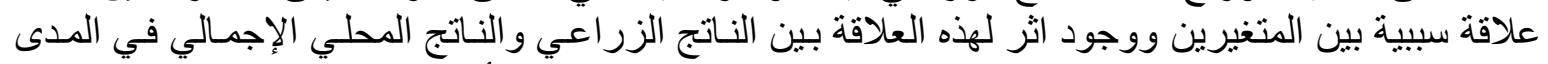

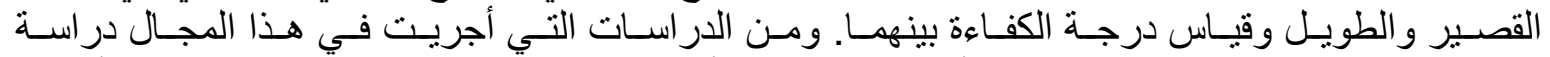
(Monadjam)

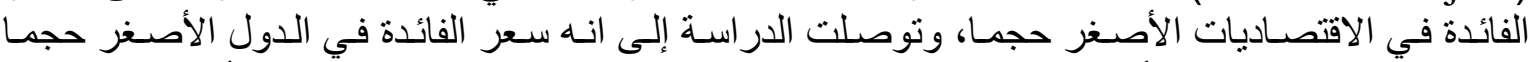

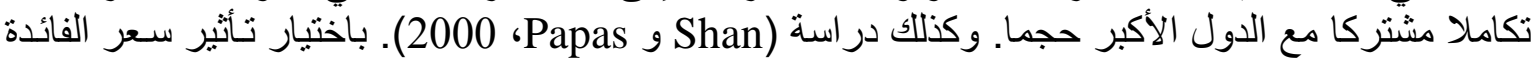

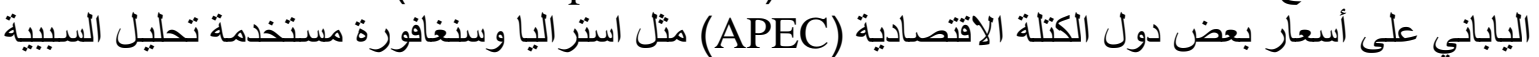

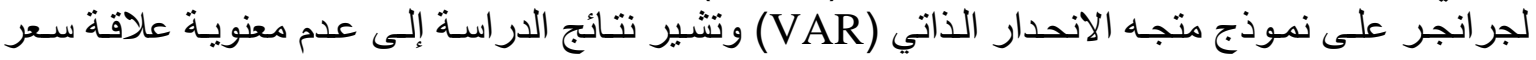

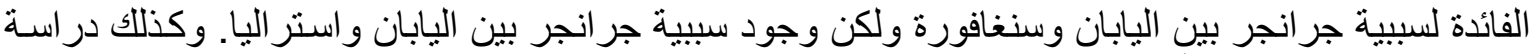

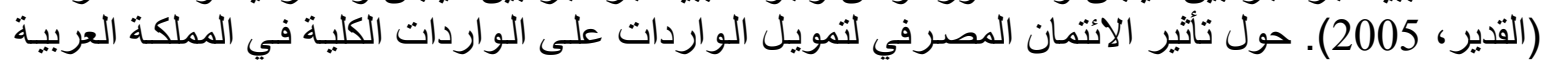

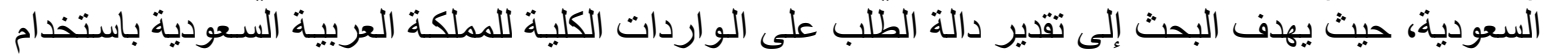

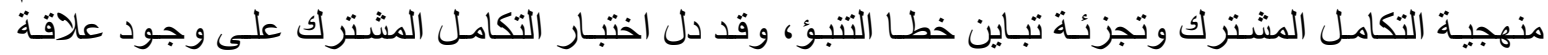

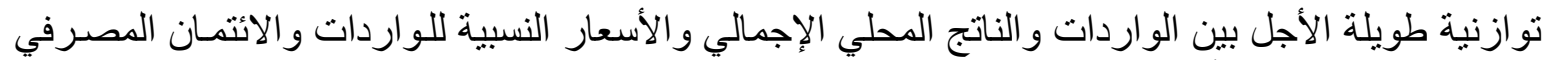

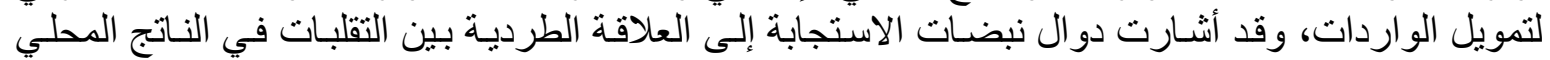

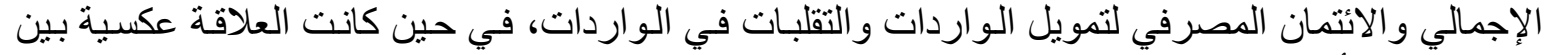

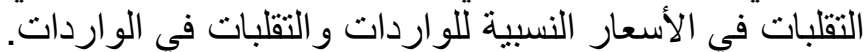

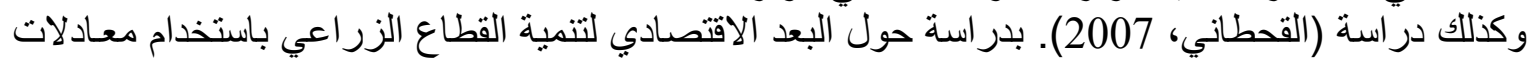

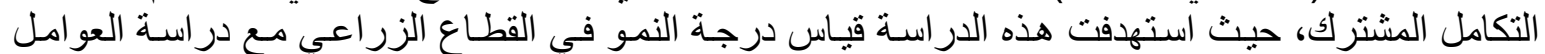

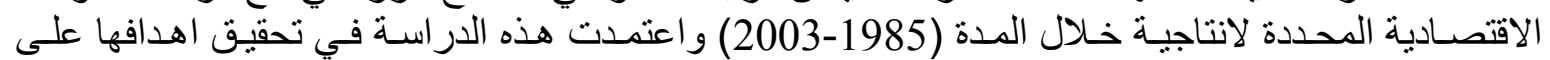

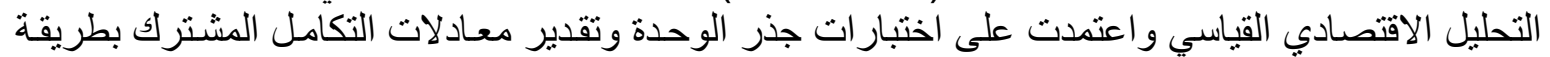

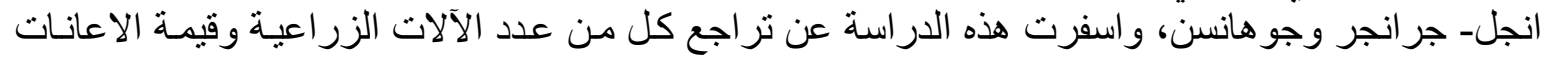

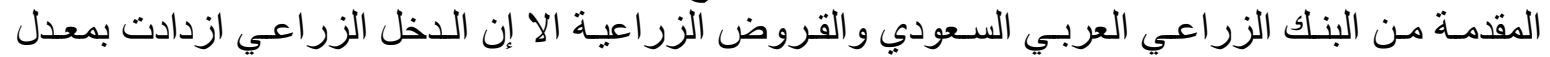
سنوي بلغ (3.1\%) خلانل مدة 1985-2003.

\section{مواد البحث وطر ائقه}

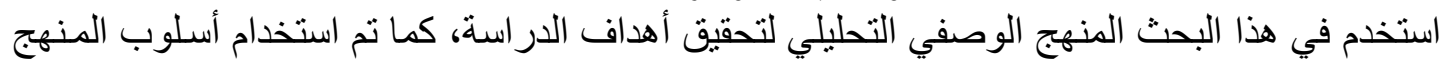

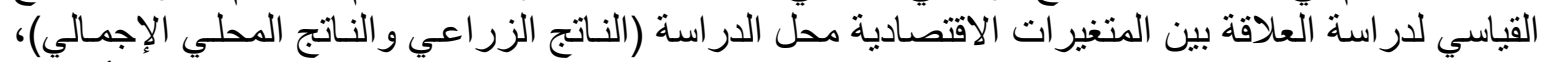

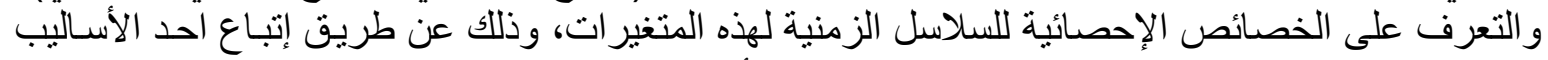

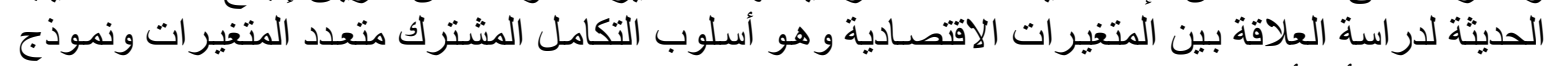

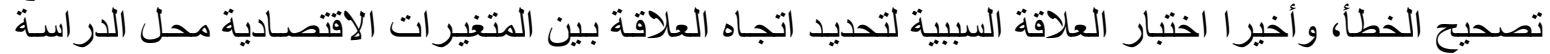

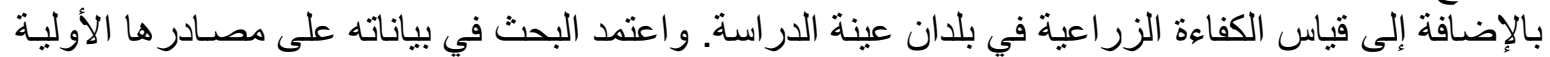

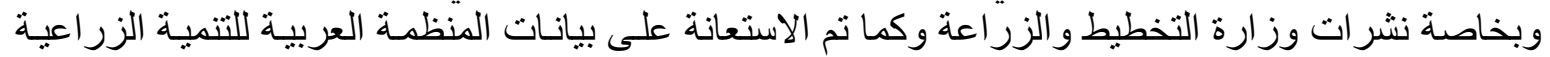

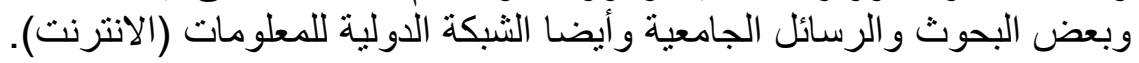




\section{النتائج والمناقشة}

يمكن بيان منهجية الدر اسة في الخطوات التالية:

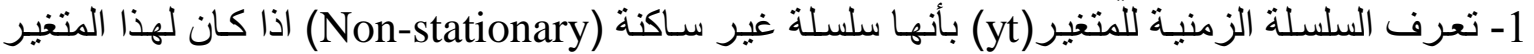

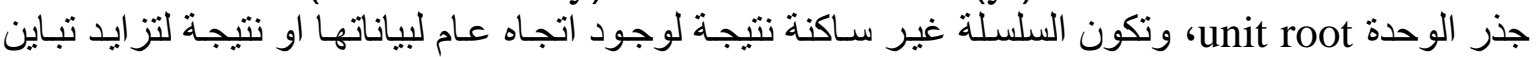

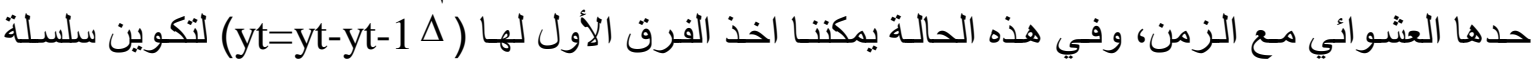
جديدة متكاملة من الدرجة الأولى (1) integrated of degree one. وتقسم السلسلة الزمنية إلى قسمين: ـ سلاسل زمنية مستقرة و المقصود بها إن قيم السلسلة تتذبذب حول وسط ثابت و لاتتاثر بتغير الزمن.

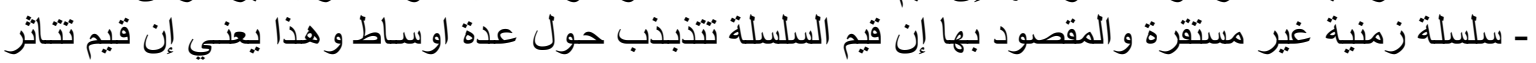

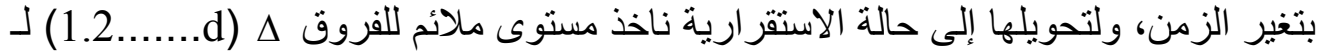

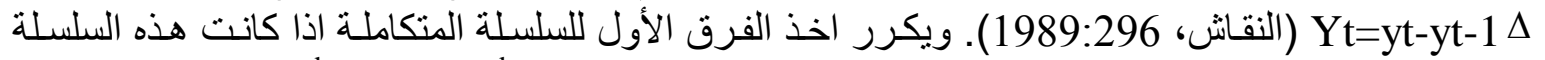

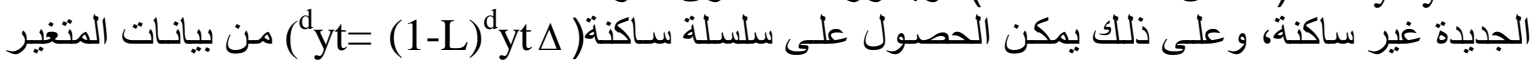

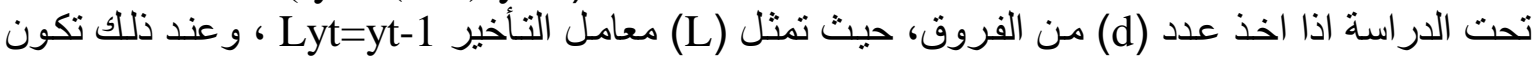

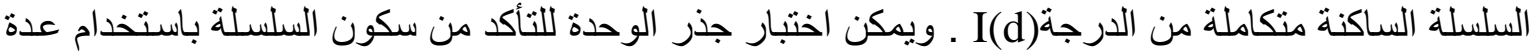

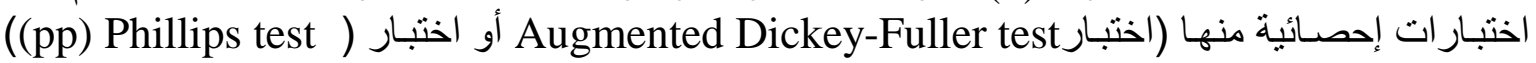
( و phillip)

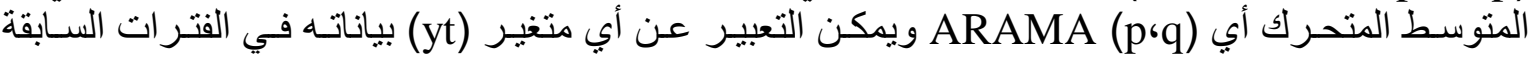
$\mathrm{Yt}=\mathrm{y} 1 \mathrm{yt}-1+\mathrm{y} 2 \mathrm{yt}-2+\ldots . . .+\mathrm{ykyt}-\mathrm{k}+\varepsilon \mathrm{t}$ بمعادلة يكون فيها الحد العشوائي مستقلا على النحو التحالي:

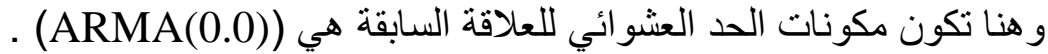

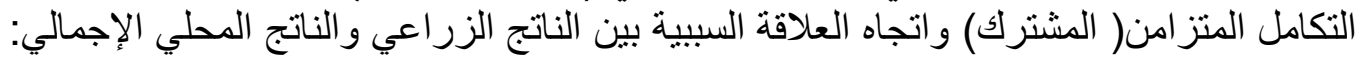

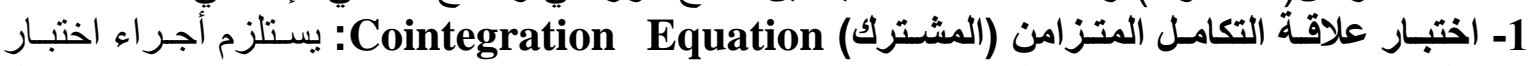

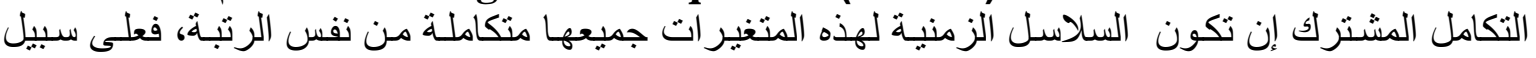

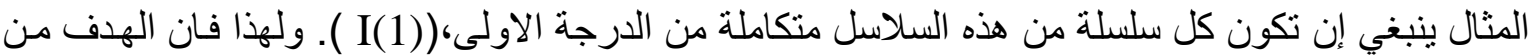

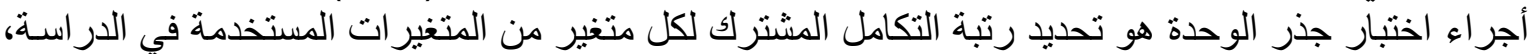

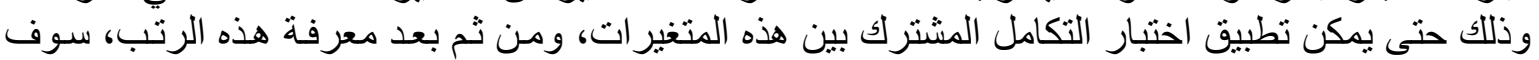

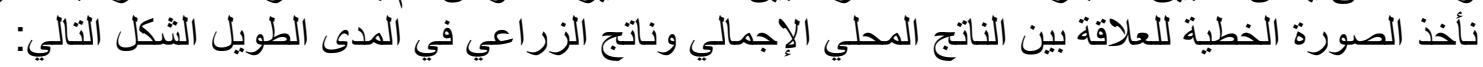
$\mathrm{Yt}=\mathrm{B} 0+\mathrm{B} 1 \mathrm{xt}$

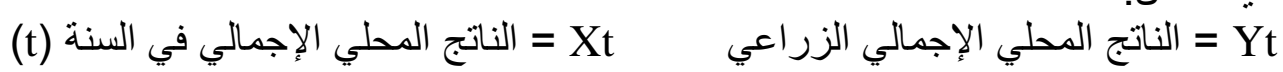

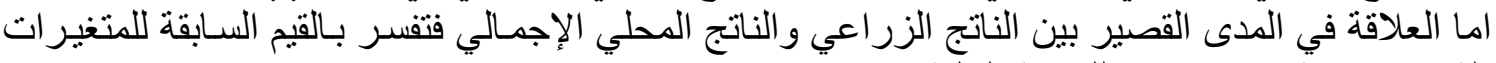

$\mathrm{Yt}=\mathrm{B} 0+\mathrm{B} 1 \mathrm{yt}-1+\mathrm{B} 2 \mathrm{xt}-1+\mathrm{ut}$ (حالة فترة سابقة واحدة) وفقا للعلاقة التالية:

\section{$\mathrm{Yt}=\mathrm{B} 0+\mathrm{B} 1 \mathrm{yt}-1+\mathrm{B} 2 \mathrm{xt}-1+\mathrm{ut}$}

حيث تمثل:

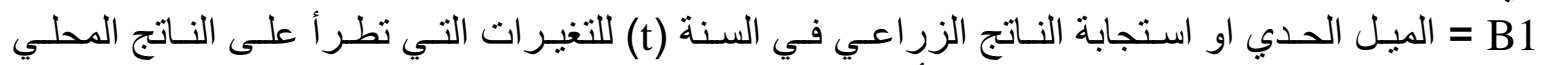
الإجمالي في السنة (t-1) ، (t) يمثل الخطأ العشو ائي في السنة (t) . المرحلة الاولى من اختبار التكامل المشترك:

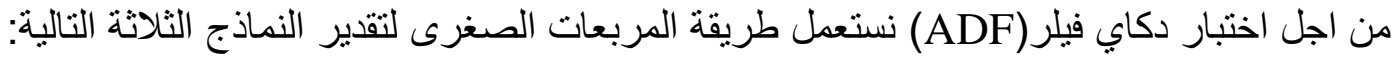

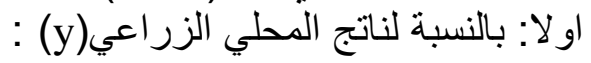

$$
\begin{aligned}
& \Delta \mathrm{yt}=\mathrm{a}^{*} \mathrm{yt}-1-\sum_{j=2}^{p} \mathrm{bj}^{*} \Delta \mathrm{yt}-\mathrm{j}+1+\varepsilon_{\mathrm{t}} \\
& \Delta \mathrm{yt}=\mathrm{a}^{*} \mathrm{yt}-1-\sum_{j=2}^{p} \mathrm{bj}^{*} \Delta \mathrm{yt}-\mathrm{j}+1+\mathrm{c}+\varepsilon_{\mathrm{t}} \\
& \Delta \mathrm{yt}=\mathrm{a}^{*} \mathrm{yt}-1-\sum_{j=2}^{p} \mathrm{bj}^{*} \Delta \mathrm{yt}-\mathrm{j}+1+\mathrm{c}+\mathrm{d}^{*} \mathrm{t}+\varepsilon_{\mathrm{t}}
\end{aligned}
$$




$$
\begin{aligned}
& \Delta \mathrm{xt}=\mathrm{a}^{*} \mathrm{xt}-1-\sum_{j=2}^{p} \mathrm{bj}^{*} \Delta \mathrm{xt}-\mathrm{j}+1+\varepsilon_{\mathrm{t}} \\
& \Delta \mathrm{xt}=\mathrm{a}^{*} \mathrm{xt}-1-\sum_{j=2}^{p} \mathrm{bj}^{*} \Delta \mathrm{xt}-\mathrm{j}+1+\mathrm{c}+\varepsilon_{\mathrm{t}} \\
& \Delta \mathrm{xt}=\mathrm{a}^{*} \mathrm{xt}-1-\sum_{j=2}^{p} \mathrm{bj}^{*} \Delta \mathrm{xt}-\mathrm{j}+1+\mathrm{c}+\mathrm{d}^{*} \mathrm{t}+\varepsilon_{\mathrm{t}}
\end{aligned}
$$

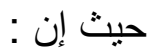

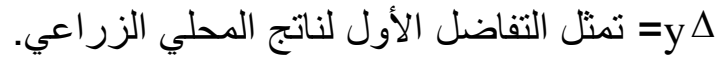

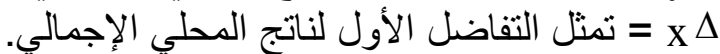

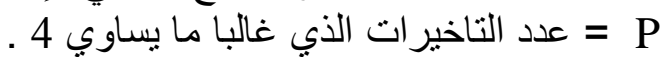
حيث يقوم اختبار دكاي فيلر ( ADF) يقوم على فرضنين bj = 1 : H0 الفرضية العدمية

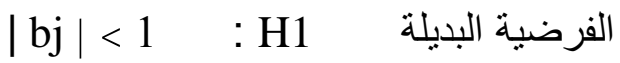

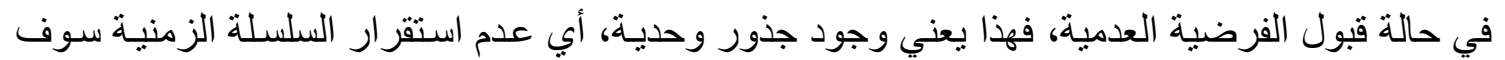

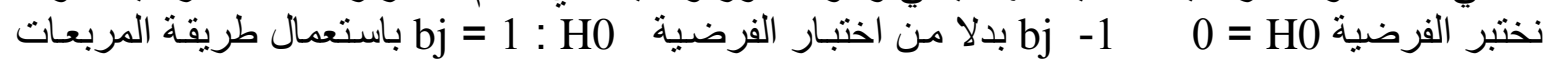

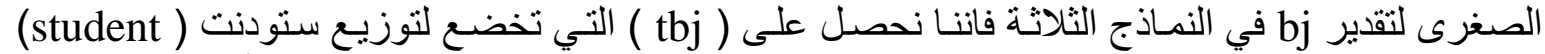

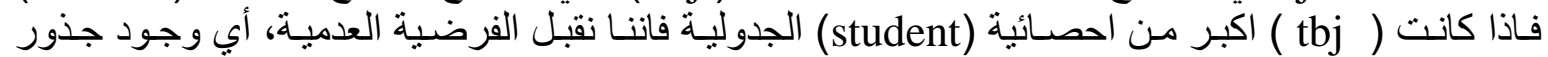
وحدية، وبالتالي عدم استقرار السلسلة الزمنية.

الجدول(1): التفاضلات الأولى للمتغيرين الناتج الزراعي والناتج المحلى الإجمالي في دول العينة.

\begin{tabular}{|c|c|c|c|c|c|}
\hline$\% 10$ & $\% 5$ & $\% 1$ & اختبار دكاي & $\begin{array}{c}\text { المتغير } \\
\text { Variable }\end{array}$ & $\begin{array}{c}\text { البلد } \\
\text { Country }\end{array}$ \\
\hline & & & 12.981- & $\mathrm{y}$ & العراق \\
\hline & & & 4.743- & $\mathrm{X}$ & Iraq \\
\hline & & & 3.307 & $\mathrm{y}$ & سعودية \\
\hline \multirow{3}{*}{$1.16-$} & \multirow{3}{*}{$1.94-$} & \multirow{3}{*}{$2.59-$} & 15.499 & $\mathrm{x}$ & Saudi \\
\hline & & & 7.658 & $\mathrm{y}$ & تونس \\
\hline & & & 12.534 & $\mathrm{x}$ & Tunisia \\
\hline
\end{tabular}
Table (1): First differentials for two variable agrarian resulting and total local resulting in sample country.

إن النتائج التي تظهر في جدول رقم (1) يتبين من خلالها بـان قيمة النـاتج الزر اعي (y) وقيمة الناتج

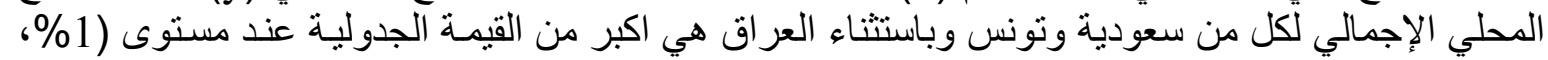

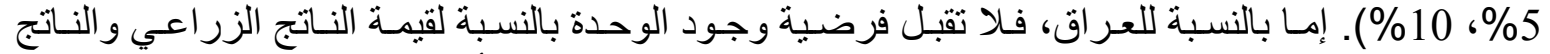

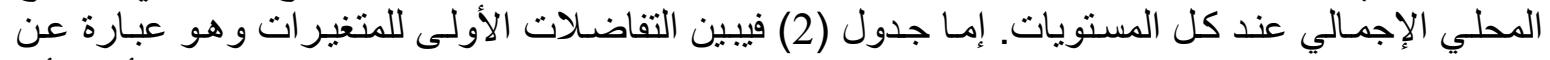
سلاسل مستقرة عند كل المستويات، وبالتالي فالسلاسل الزمنية لكل المتغيرات متكاملة من الدرجة الأولى الألى أي

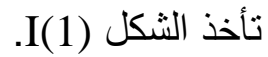

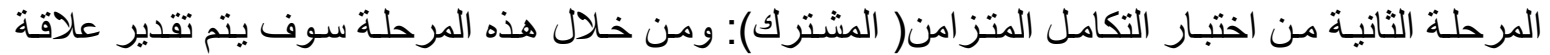

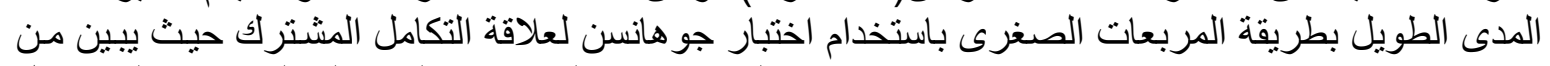

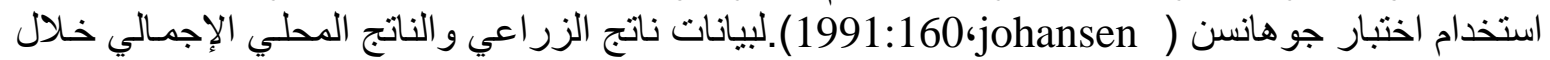

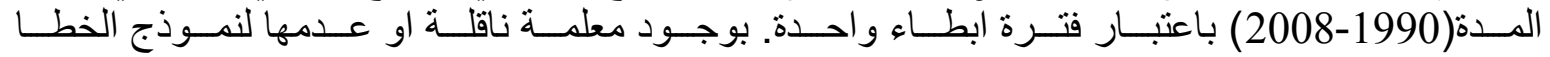

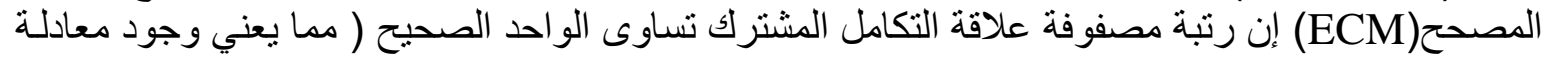
واحدة للعلاقة في الددى الطويل بين المتغيرين). 
Mesopotamia J. of Agric.

Vol. (41) No. (4) 2013
مـــــلـة زر اعـــة الــر افـدين المجلد (41) العدد (4) 2013

الجدول (2): التفاضـلات الأولى في التغير للمتغيرين النـاتج الزراعي و النـاتج المحلى الإجمـالي في دول

Table (2): First differentials in change for two variable agrarian resulting and total local resulting in sample country.

\begin{tabular}{|c|c|c|c|c|c|}
\hline$\% 10$ & $\% 5$ & $\% 1$ & فتبلر دكاي & $\begin{array}{c}\text { المتغير } \\
\text { variable }\end{array}$ & $\begin{array}{c}\text { البلد } \\
\text { Country }\end{array}$ \\
\hline & & & $5.273-$ & $\mathrm{y} \Delta$ & العراق \\
\hline & & & 1.197- & $\mathrm{x} \Delta$ & Iraq \\
\hline & & & $0.454-$ & y $\Delta$ & \multirow{2}{*}{$\begin{array}{l}\text { سعودية } \\
\text { Saudi }\end{array}$} \\
\hline \multirow{3}{*}{$1.16-$} & \multirow{3}{*}{$1.94-$} & \multirow{3}{*}{$2.59-$} & 0.312 & $\mathrm{x} \Delta$ & \\
\hline & & & $0.729-$ & $\mathrm{y} \Delta$ & \multirow{2}{*}{$\begin{array}{c}\text { تونس } \\
\text { Tunisia }\end{array}$} \\
\hline & & & $0.401-$ & $\mathrm{x} \Delta$ & \\
\hline
\end{tabular}

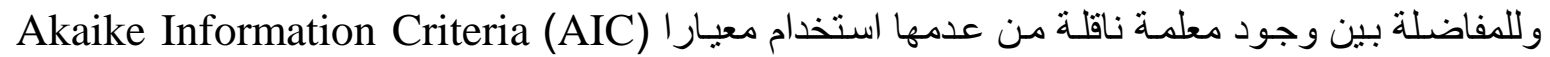

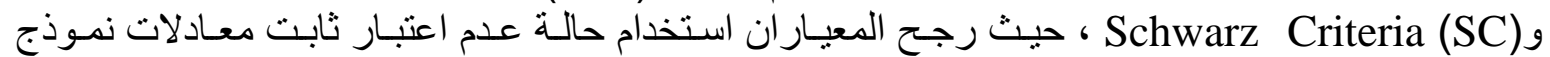
(ECM)

$\mathrm{Y}=-90.99+2.693 \mathrm{x}$

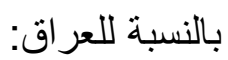

$\mathrm{T}=(-4.090)(15.29)$

$\mathrm{R}^{2}=0.932 \quad \mathrm{AIC}=189.360 \quad \mathrm{SC}=192.193$

$\mathrm{Y}=120.602+0.164 \mathrm{x}$

وبالنسبة للسعودية:

$\mathrm{T}=(18.86) \quad(7.019)$

$\mathrm{R}^{2}=0.743 \quad \mathrm{AIC}=122.119 \quad \mathrm{SC}=124.952$

$\mathrm{Y}=85.538+0.603 \mathrm{x}$

وبالنسبة لتونس:

$\mathrm{T}=(5.814) \quad$ (9.9)

$\mathrm{R}^{2}=0.854 \quad \mathrm{AIC}=168.416 \quad \mathrm{SC}=171.249$

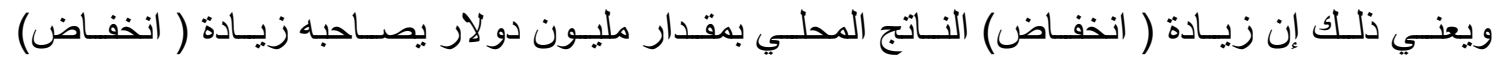

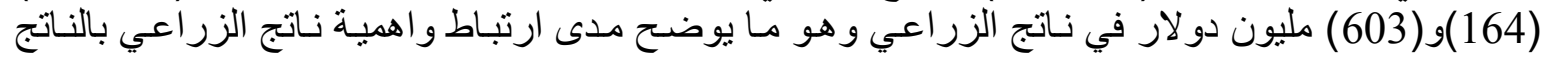

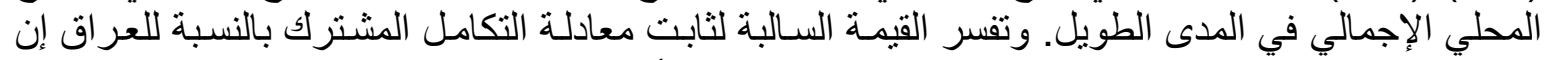

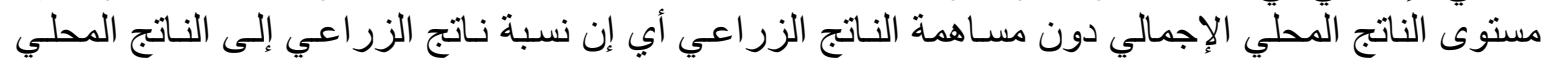

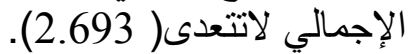

2- اختبار اتجاه العلاقة السببية Causality Test: سنحاول في هذه المرحلة اختبار اتجاه العلاقة السببية

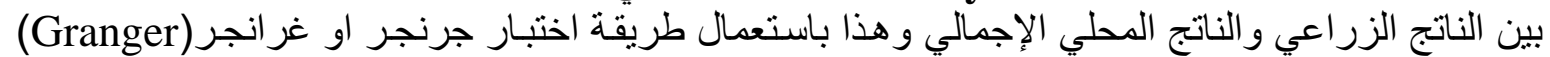

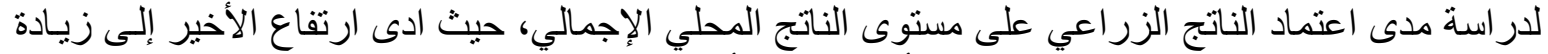

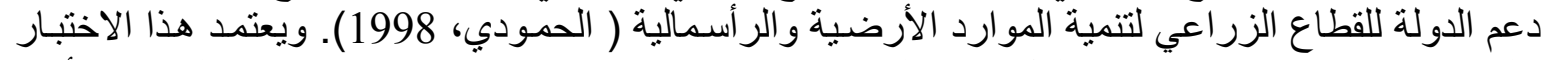

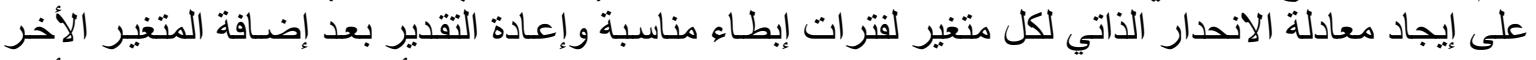

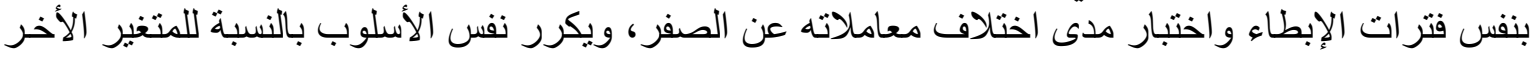
ويركز اختبار جرانجر (Granger)،

$\Delta \mathrm{yt}=\sum_{i=1}^{n} \mathrm{a}^{*} \Delta \mathrm{xt}-\mathrm{I}+\varepsilon_{\mathrm{t}}$ 


$$
\begin{aligned}
& \Delta \mathrm{xt}=\sum_{i=1}^{n} \mathrm{bi}^{*} \Delta \mathrm{yt}-\mathrm{I}+\varepsilon_{\mathrm{t}} \ldots \ldots \ldots \ldots \ldots \ldots \ldots \ldots \ldots \ldots \\
& \Delta \mathrm{yt}=\sum_{i=1}^{n} \mathrm{ci}^{*} \Delta \mathrm{yt}-\mathrm{I}+\sum_{i=1}^{n} \mathrm{di}^{*} \Delta \mathrm{xt}-\mathrm{I}+\varepsilon_{\mathrm{t}} \\
& \Delta \mathrm{xt}=\sum_{i=1}^{n} \mathrm{ei}^{*} \Delta \mathrm{xt}-\mathrm{I}+\sum_{i=1}^{n} \mathrm{hi}^{*} \Delta \mathrm{yt}-\mathrm{I}+\varepsilon_{\mathrm{t}}
\end{aligned}
$$

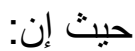
y

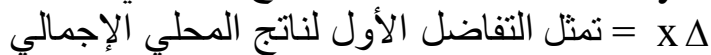

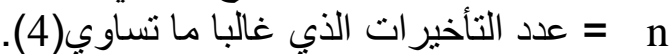

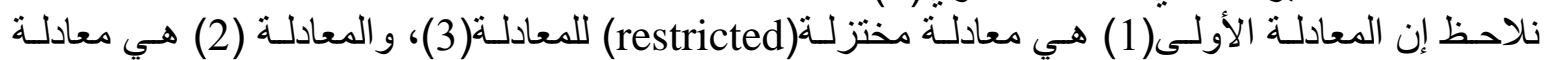
مختزلة(restricted) للمعادلة(4)، لاختيار العلاقة السبيبة، سوف نستعمل الفرضيتين التاليتين:

$$
\text { hi=0 = H0 } \mathrm{di}=0
$$

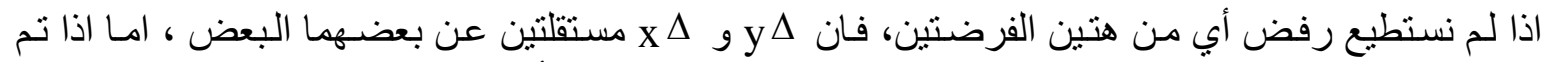

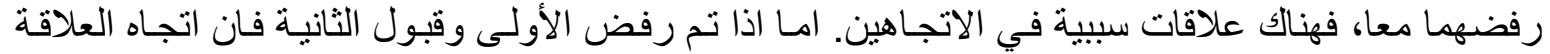

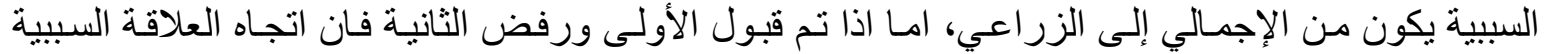

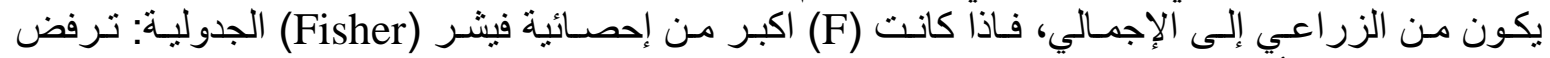

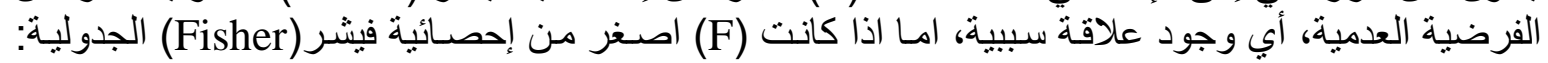
تقبل الفرضية العدمية، أي عدم وجود علية علاقة السبيية. لاينا قيم الجدولية لاحصائية فيشر (Fisher) في هذه الحالة الحية كما يلي:

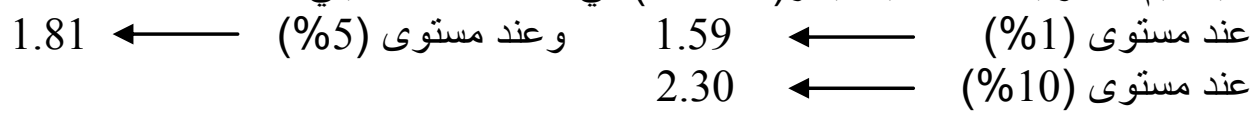

الجدول(3): يبين القيم الجدولية لاحصائية فيشر(Fisher).

Table (3): Show values schedule for statistical fisher (Fisher).

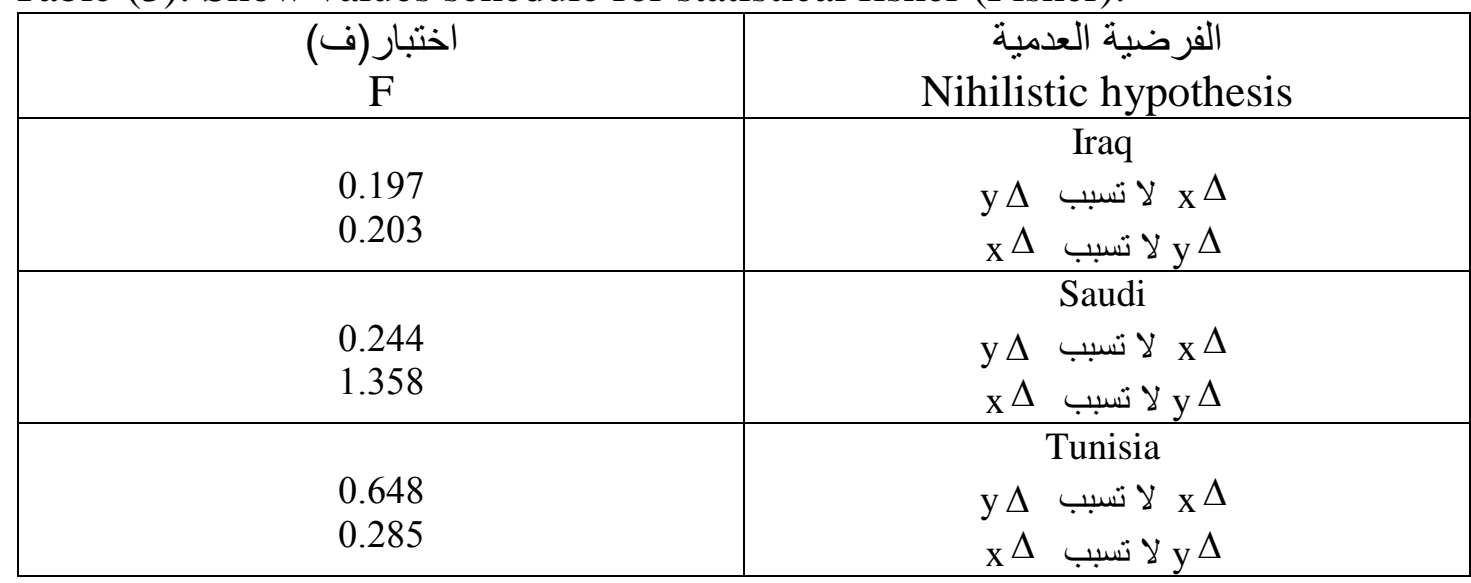

من الجدول (3)، نلاحظ إن كل قيم (F) المحسوبة هي اقل من احصائية فيشر(Fisher) الجدولية، وهذا

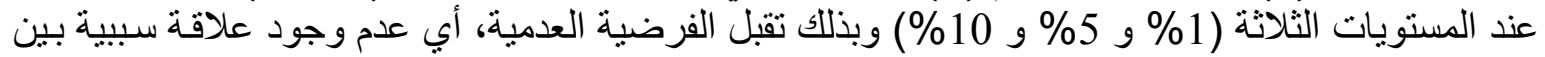
الناتج الزر اعي و الناتج المحلي الإجمالي في كل من العر اق وسعودية ونونس.

3ـ قياس الكفاءة الزراعية :Agricultural Efficiency: لقد تم اعتماد المعادلة التالية في حساب درجة $\mathrm{E}=\mathrm{rad} / \mathrm{rat}$ 
Mesopotamia J. of Agric.

Vol. (41) No. (4) 2013
ISSN:2224-9796(Online)

ISSN: 1815-316x (Print)
مــــــلة زر اعـــة الــر افــدين

المجلد (41) العدد (4) 2013

= نسبة مساهمة الناتج الزراعي في الناتج المحلي الإجمالي. rad rat

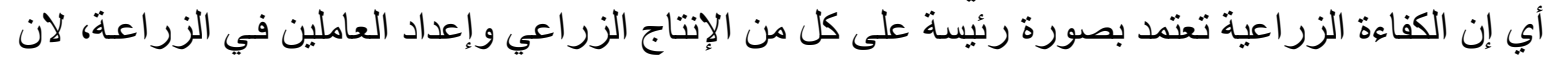

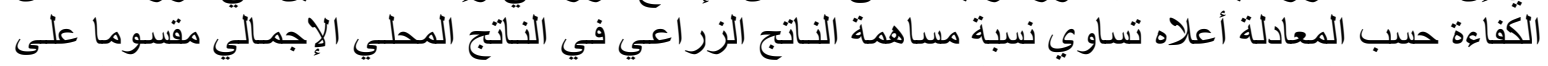

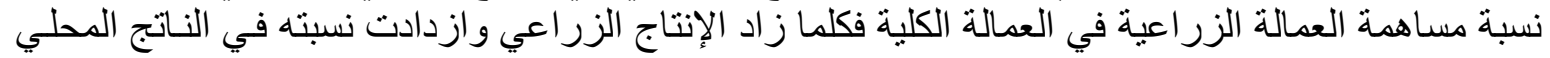

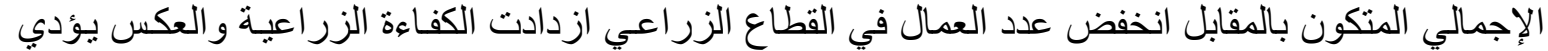

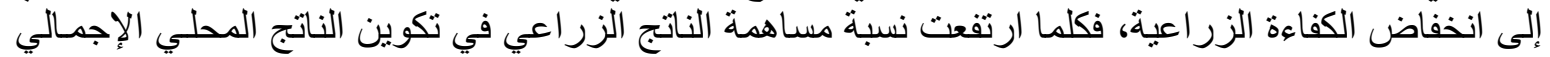

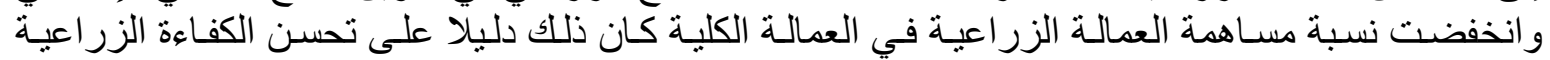

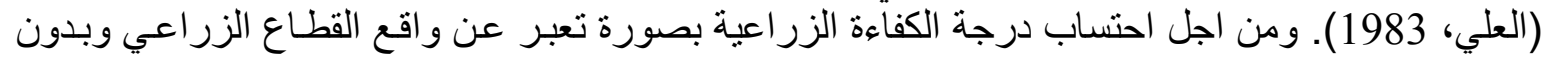

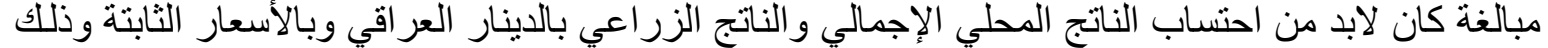

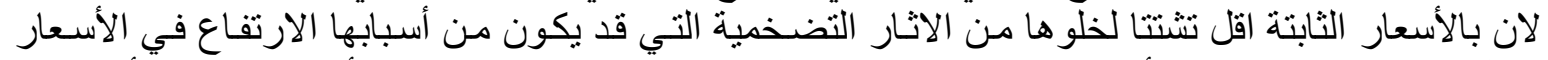

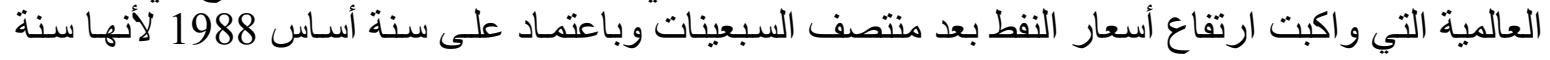

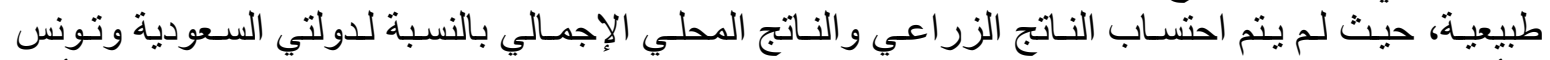

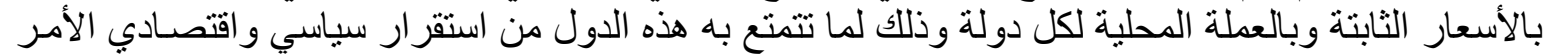

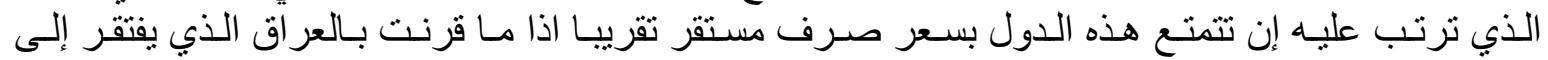
الاستقر ار في جميع النواحي، ومن خلان هذه النظر إلى الجدول (4):

الجدول (4): الكفاءة الزر اعيـة ونسبة مسـاهمة النـاتج الزراعي في النـاتج المحلي الاجمـالي بالاسـعار الثابتـة

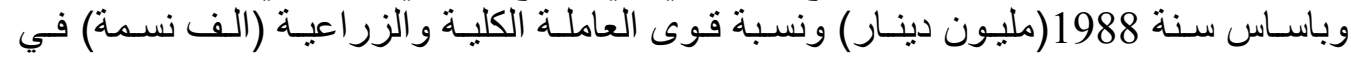

Table (4): Agricultural efficiency and ratio sharing agrarian resulting and total local resulting for constant prices and foundation year 1988 (million dinar) and ratio forces total labor and agricultural (person 1000) in Iraq.

\begin{tabular}{|c|c|c|c|}
\hline $\begin{array}{c}\text { الكفاءة الزراعية } \\
\text { Agricultural } \\
\text { efficiency }\end{array}$ & $\begin{array}{c}\text { نسبة مساهمة العمالة الزر اعية الى العلية } \\
\text { Ratio forces labor } \\
\text { Ratic } \\
\text { agricultural to total labor }\end{array}$ & 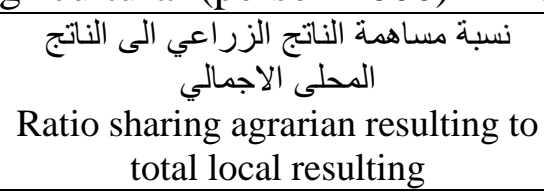 & $\begin{array}{l}\text { السنوات } \\
\text { Years }\end{array}$ \\
\hline 0.44 & 26.03 & 11.60 & 1990 \\
\hline 1.01 & 26.51 & 26.93 & 1991 \\
\hline 0.94 & 26.51 & 24.93 & 1992 \\
\hline 0.71 & 26.52 & 18.92 & 1993 \\
\hline 0.73 & 26.51 & 19.52 & 1994 \\
\hline 0.80 & 26.49 & 21.39 & 1995 \\
\hline 0.78 & 26.49 & 20.70 & 1996 \\
\hline 0.88 & 17.73 & 15.69 & 1997 \\
\hline 0.71 & 17.71 & 12.593 & 1998 \\
\hline 0.88 & 17.73 & 12.42 & 1999 \\
\hline 0.61 & 17.72 & 10.83 & 2000 \\
\hline 0.60 & 17.72 & 10.71 & 2001 \\
\hline 0.73 & 18.30 & 13.46 & 2002 \\
\hline 0.75 & 18.86 & 14.26 & 2003 \\
\hline 0.60 & 18.86 & 11.40 & 2004 \\
\hline 0.60 & 18.85 & 11.44 & 2005 \\
\hline 0.71 & 19.16 & 13.67 & 2006 \\
\hline 0.81 & 19.17 & 15.53 & 2007 \\
\hline 0.66 & 19.18 & 12.78 & 2008 \\
\hline
\end{tabular}

المصدر: احتسب الجدول بالاعتماد على بيانات التالية: مجهول (2005) ، الكتاب السنوي للإحصاءات الزر اعية لسنوات مختلفة

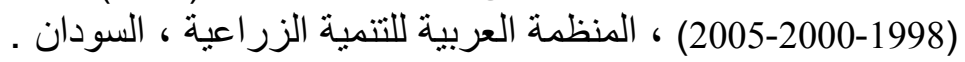

(2005) , yearbook for statistics agricultural for different years (1998- 2000-2005) , organization arabic for development agricultural, Sudan . 
Mesopotamia J. of Agric.

Vol. (41) No. (4) 2013
ISSN:2224-9796(Online)

ISSN: 1815-316x (Print)
مـــــلة زر اعـــة الـرافـدين المجلد (41) العدد (4) 2013

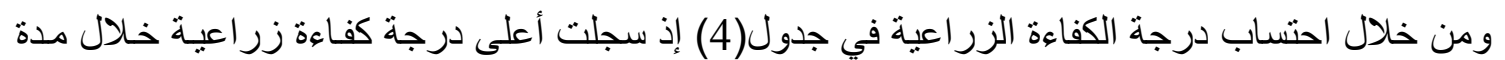

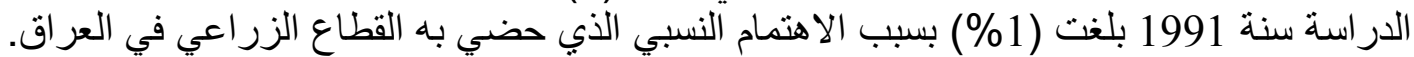

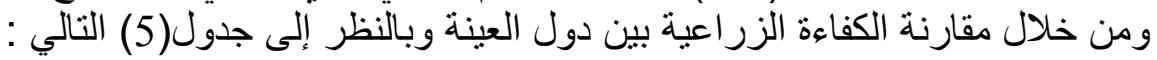

الجدول (5): الكفاءة الزراعية ونسبة مساهمة الناتج الزراعي في النـاتج المحلي الإجمـالي بالأسعار الجاريـة (مليون دو لار) ونسبة قوى العاملة الكلية والزر اعية اعية (الف نسمة) في سعودية.

Table (5): Agricultural efficiency and ratio sharing agrarian resulting and total local resulting for current prices (million dollar) and ratio forces total labor and agricultural (person 1000) in Saudi .

\begin{tabular}{|c|c|c|c|}
\hline $\begin{array}{c}\text { Agricultural } \\
\text { efficiency }\end{array}$ & $\begin{array}{c}\text { الزربة مساهمة العية الى العمالة الكملة } \\
\text { Ratio forces labor } \\
\text { الكية } \\
\text { agricultural to total } \\
\text { labor }\end{array}$ & $\begin{array}{c}\text { نسبة مساهمة الناتج الزراعي الى الناتج الاجمالي } \\
\text { Ratio sharing } \\
\text { Rarian resulting } \\
\text { agrarian } \\
\text { to total local } \\
\text { resulting }\end{array}$ & $\begin{array}{l}\text { السنوات } \\
\text { Years }\end{array}$ \\
\hline 0.16 & 39.19 & 6.53 & 1990 \\
\hline 0.16 & 38.31 & 6.26 & 1991 \\
\hline 0.17 & 37.46 & 6.51 & 1992 \\
\hline 0.19 & 35.99 & 7.04 & 1993 \\
\hline 0.19 & 34.57 & 6.58 & 1994 \\
\hline 0.45 & 15.16 & 6.84 & 1995 \\
\hline 0.43 & 14.70 & 6.40 & 1996 \\
\hline 0.43 & 14.25 & 6.15 & 1997 \\
\hline 1.06 & 6.64 & 7.05 & 1998 \\
\hline 0.77 & 7.77 & 6.01 & 1999 \\
\hline 0.63 & 7.76 & 4.94 & 2000 \\
\hline 0.67 & 7.69 & 5.20 & 2001 \\
\hline 0.65 & 7.76 & 5.11 & 2002 \\
\hline 0.23 & 19.60 & 4.53 & 2003 \\
\hline 0.24 & 16.32 & 3.95 & 2004 \\
\hline 0.20 & 16.30 & 3.31 & 2005 \\
\hline 0.19 & 18.51 & 3.53 & 2006 \\
\hline 0.35 & 16.71 & 5.91 & 2007 \\
\hline 0.34 & 16.88 & 5.89 & 2008 \\
\hline
\end{tabular}

الدصدر: احتسب الجدول بالاعتماد على بيانات التالية: مجهول (2005) ، الكتاب السنوي للإحصاءات الزر اعية لسنوات مختلفة

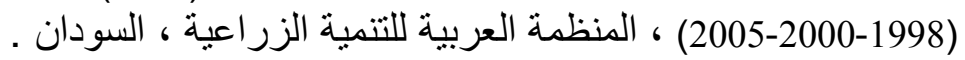
(2005) , yearbook for statistics agricultural for different years (1998- 2000-2005), organization arabic for development agricultural, Sudan .

وبالنظر الى الجدول (5) نجد إن السعودية قد سجلت اعلى درجة كفاءة زر اعية خلال مدة الدر اسة سنة 1998

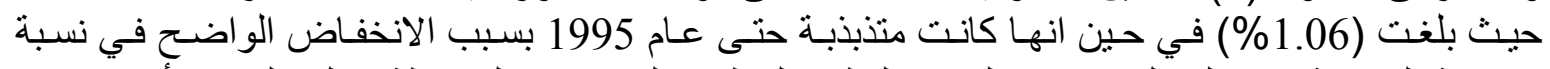

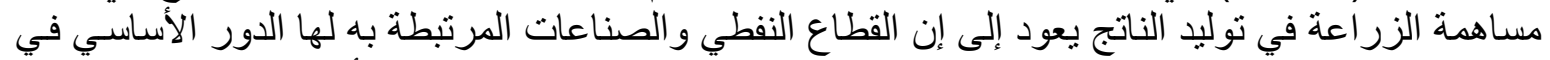

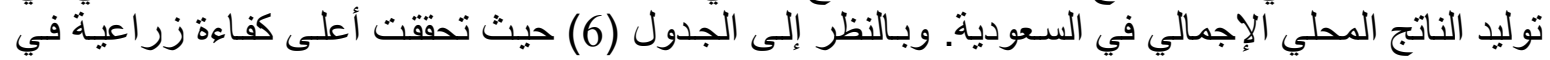

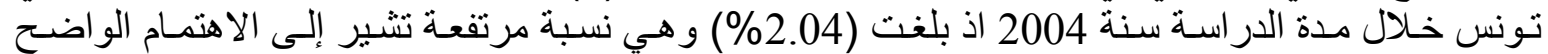

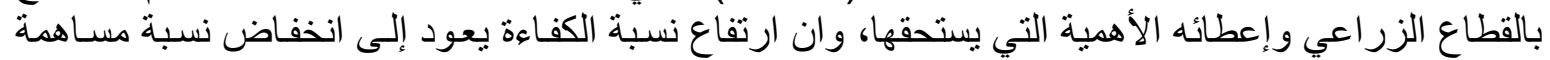


Mesopotamia J. of Agric.

Vol. (41) No. (4) 2013

العمالة الزر اعية في العمالة الكلية ويرجع هذا الانخفاض نتيجة لاستخدام مستلزمات الإنتاج الحديثة واستخدام

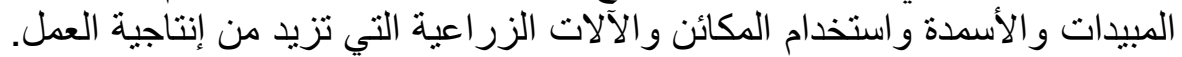

الجدول (6): الكفاءة الزراعية ونسبة مساهمة الناتج الزر اعي في النـاتج المحلي الاجمـالي بالاسعار الجاريـة

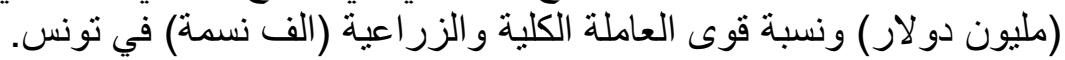

Table (6): Agricultural efficiency and ratio sharing agrarian resulting and total local resulting for current prices (million dollar) and ratio forces total labor and agricultural (person 1000) in Tunisia.

\begin{tabular}{|c|c|c|c|}
\hline $\begin{array}{c}\text { الكفاءة الزر اعية } \\
\text { Agricultural } \\
\text { efficiency }\end{array}$ & $\begin{array}{l}\text { الزربة مساهمة الكية الى العمالة } \\
\text { الكمالة } \\
\text { Ratio forces labor } \\
\text { agricultural to } \\
\text { total labor }\end{array}$ & $\begin{array}{c}\text { نسبة مساهمة الناتج الزراعي اللى الناتج الاجمالي } \\
\text { Ratio sharing } \\
\text { المحلى } \\
\text { agrarian resulting to } \\
\text { total local resulting }\end{array}$ & $\begin{array}{l}\text { السنوات } \\
\text { Years }\end{array}$ \\
\hline 0.67 & 24.29 & 16.30 & 1990 \\
\hline 0.76 & 23.35 & 17.97 & 1991 \\
\hline 0.78 & 22.38 & 17.65 & 1992 \\
\hline 0.55 & 33.26 & 18.33 & 1993 \\
\hline 0.49 & 30.93 & 15.40 & 1994 \\
\hline 0.42 & 30.60 & 13.07 & 1995 \\
\hline 0.56 & 27.83 & 15.65 & 1996 \\
\hline 0.53 & 27.83 & 15.02 & 1997 \\
\hline 0.60 & 20.68 & 12.46 & 1998 \\
\hline 0.61 & 20.10 & 12.80 & 1999 \\
\hline 0.61 & 21.87 & 12.35 & 2000 \\
\hline 0.55 & 21.03 & 11.63 & 2001 \\
\hline 0.49 & 6.08 & 10.32 & 2002 \\
\hline 1.97 & 6.14 & 12.04 & 2003 \\
\hline 2.04 & 5.97 & 12.58 & 2004 \\
\hline 1.98 & 5.87 & 11.64 & 2005 \\
\hline 1.71 & 6.70 & 11.51 & 2006 \\
\hline 1.92 & 6.78 & 13.04 & 2007 \\
\hline 1.68 & 7.80 & 13.14 & 2008 \\
\hline
\end{tabular}

احتسب الجدول بالاعتماد على بيانات التالية: مجهول (2005) ، الكتاب السنوي للإحصاءات الزر اعية لسنوات مختلفة (1998-

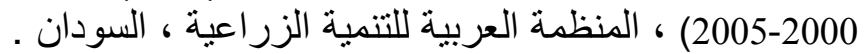

(2005) , yearbook for statistics agricultural for different years (1998- 2000-2005) , organization arabic for development agricultural, Sudan .

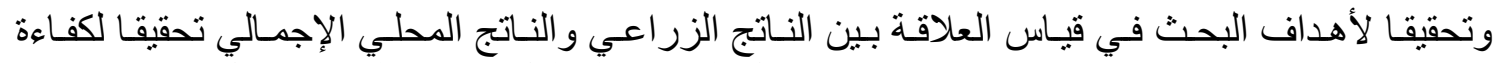

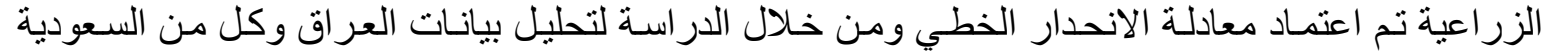

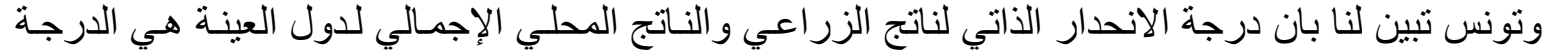

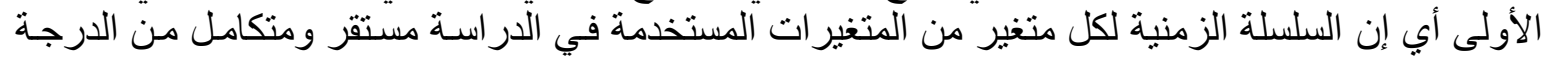

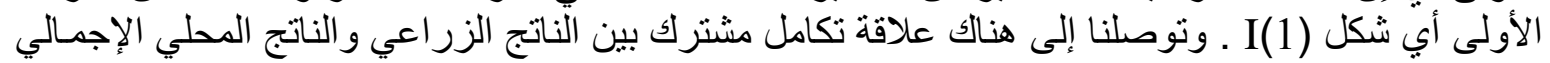

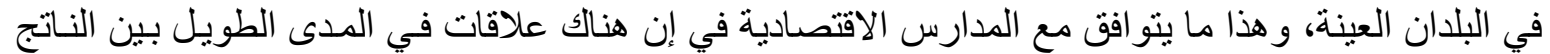

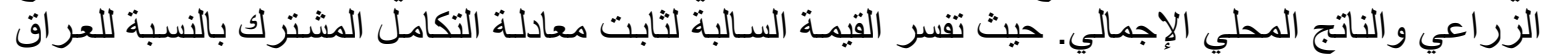

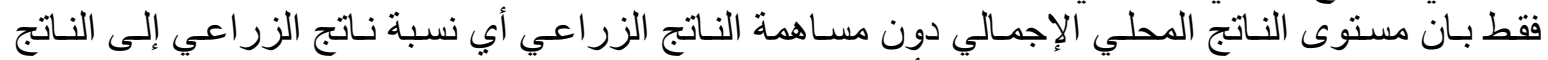
المحلي الإجمالي لاتتعدى (2.693). ويتبين أيضا من الدر اسة عدم وجود علاقة سبيية بين المتغيرين في في كل الني 


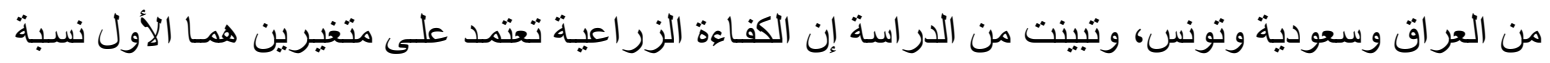

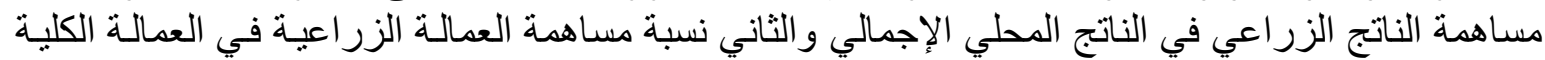

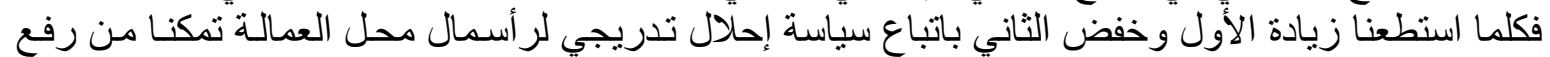

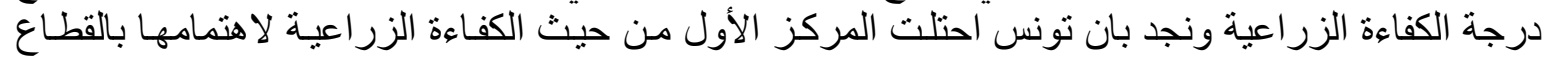

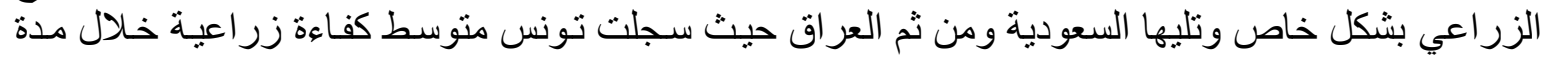

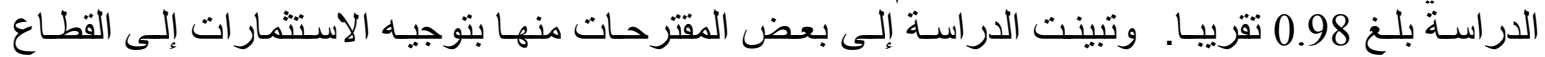

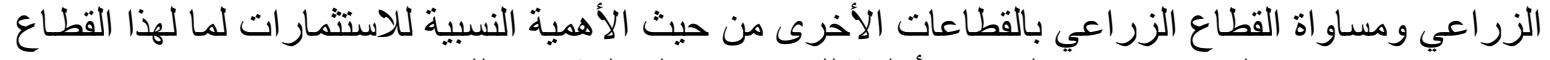

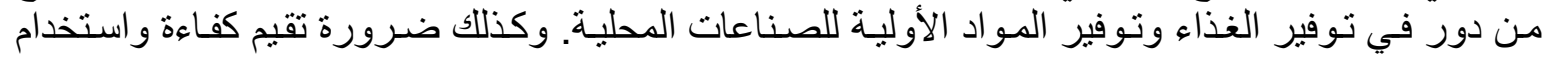

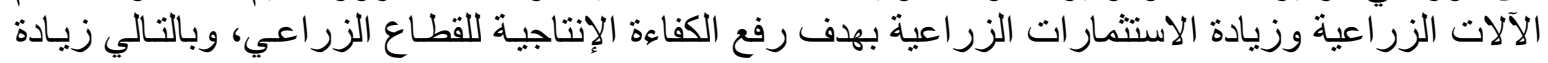
نصيبه النسبي في الدخل المحلي الإجمالي.

\title{
COINTEGRATION EQUATION DECELERATION TIME TO MEASURE THE RELATIONSHIP BETWEEN AGRICULTURAL OUTPUT AND GDP INVESTIGATION "OF AGRICULTURAL EFFICIENYCY IN THE SELECTED DEVELOPING COUNTRIES ( IRAQ - SAUDI ARABIA - TUNISIA \\ DURING (1990-2008)
}

Lora B. Basher AI-Saor

Econ.dept., college of Agric. And Foresty, Mosul Univ.,Iraq.

Email : Lora.basim@yahoo.com

\begin{abstract}
The study aims to analyses the relationship between agrarian resulting and total local resulting. Iraq, Saudi, Tunisia takes as applicative state. This research depended on the yearly data of (1990-2008) and on theoretic foundation of relationship between agrarian resulting and total local resulting there extrusive relation and agrarian resulting effect on the total local resulting, that appear throughout the causality analysis of granger concept, there is no duple causality relation between both resulting where each affects on anther. The results appear that the agrarian efficiency depend on two variable the first rate agrarian resulting sharing on the total local resulting and the second rate agrarian force sharing on the total force. Its found through this efficiency, that Tunisia is the first throught the agrarian efficiency because attending with agrarian sector specifically where agrarian efficiency medial standard through of (1990-2008) amount nearly (0.98). the stud recommend to evaluate efficiency of agricultural investment and machinery use in order to increase productivity efficiency of agricultural sector, and hence to increase its share in gross national income.
\end{abstract}

Key words: cointegration, Agricultural Efficiency, Total local resulting.

Received:20/11/2011 Accepted: 12/5/2011.

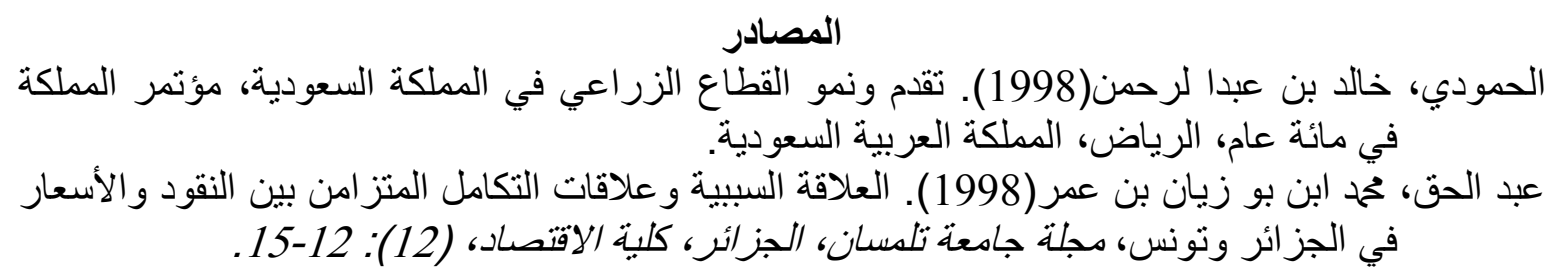


العلي، احمد بريهي (1983). أساليب التخطيط العام (الجزء الأول)، وزارة التخطيط، المعهد القومي

القحطاني، سفر بن حسين و غانم، عادل محمد خليفة (2007). البعد الاقتصادي لتنمية القطاع الزر اعي باستخدام

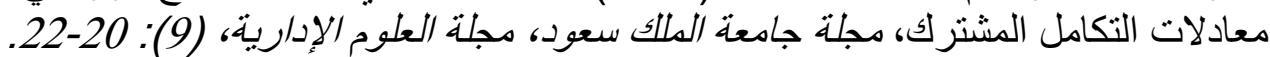

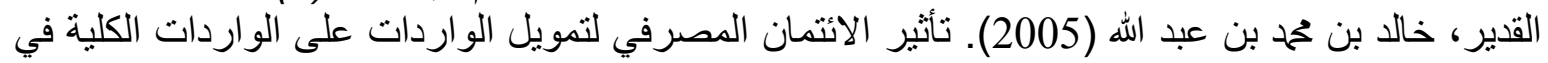

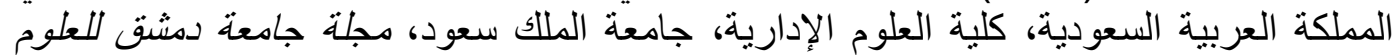

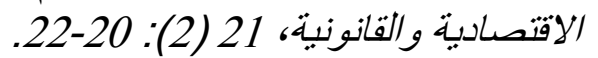

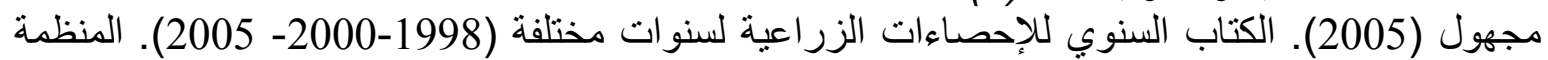

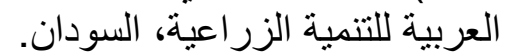

النقاش، افتخار عبدالحميد (1989). تحليل السلاسل الزمنية للتنبؤ بزر اعة الحنطة في العراق، مجلة تنمية الر افدين، (27): 296.

Granger, C. W. J. (1969). Investigating causal relations by econometris models and cross-spectral methods. Econometrica , 37:424-438.

Johansen S (1991). Estimation and Hypothesis Testing of Cointegration Vectors In Gaussian Vector, Book Store, U. S .A: 160.

Monadjem, M. (1998).Are real interest rates cointegrated. A study of three OECD countries, Applied Economics Letter, 5: 681-683.

Phillips, P. C. B and P. Perron (1988). Testing for unit root in time series regression Biometrika, 75: 335-346.

Shan, j. and Pappas, N (2000). The relative impacts of Japanese and US interest rates on local interest rates in Australia and Singapore: Granger causality test. Applied Financial Economics, 10: 291-298.

Yiannis. P. Venieris and D. Scebold (1977). Macroeconomics Models and Policy .Oregon State University, Book Store, U.S.A. 